\title{
Reflexiones en torno al pensamiento del profesor ${ }^{*}$
}

\author{
Wilson Fernando Gualteros Garnica* \\ Miguel Ángel Córdoba Barbosa ${ }^{* * *}$
}

\begin{abstract}
Gualteros G., W. F. y Cordoba B., M. A. (2010). Reflexiones en torno al pensamiento del profesor. Revista Activos, 15, 15-32.
\end{abstract}

Recibido: 7 de septiembre de 2010 Aprobado: 26 de noviembre de 2010

\section{Resumen}

En el presente artículo se hace una reflexión de la forma como el profesor construye conocimiento, cómo su forma de enseñar depende no sólo del conocimiento profesional sino del contexto y de su historia de vida. Para el efecto se plantea una visión alrededor de la investigación del pensamiento del profesor, el sentido de la reflexión en la acción y su relación con el conocimiento y las teorías emergentes.

Se inicia desde la visión de la enseñanza a partir de una concepción de conocimiento ligado a la acción, mostrando lo referente a qué conocen los profesores y cómo llegan a conocerlo; desde esta mirada, se plantean algunas categorías básicas que deben considerarse en el proceso analítico, tales como: pensamientos durante la enseñanza interactiva, creencias y teorías del profesor.

* Artículo de reflexión y comprensión contable.

** Contador Público, Magíster en Educación, Profesor de tiempo completo de la Facultad de Contaduría Pública de la Universidad Santo Tomás, Bogotá.

*** Licenciado en Psicología y Pedagogía, Magíster en Educación, Profesor Universidad Central de Bogotá. 


\title{
Palabras clave
}

Conocimiento profesional, pensamiento del profesor, complejidad, epistemología, acción Gualteros G., W. F. and Córdoba B., M. A. (2010). Reflections on teacher thinking. Activos Review, 15, 15-32.

\begin{abstract}
In this paper, a reflection on the way teachers construct knowledge, how their teaching depends not only on professional knowledge but on the context and history of life. For this purpose a vision is risen about the investigation of teacher thinking, a sense of reflection in action and its relationship with the knowledge and emerging theories.

It starts from the view of teaching from a conception of knowledge linked to action, showing respect to what teachers know and how they come to know, from this view, some basic categories are risen to be considered in the analytical process such as thoughts during interactive teaching, teacher beliefs and theories.
\end{abstract}

\section{Keywords}

Professional knowledge, teacher thinking, complexity, epistemology, action

\section{Gualteros G., W. F. et Córdoba B., M. A. (2010). Réflexions autour de la pensée du professeur. Revue Activos, 15, 15-32.}

\section{Résumé}

Le présent article présente une réflexion sur la manière dont le professeur construit la connaissance et sur sa manière d’enseigner, qui dépend non seulement de sa connaissance professionnelle mais aussi du contexte et de son histoire de vie. C'est dans cette idée que s' envisage la recherche sur la pensée du professeur, le sens de la réflexion dans l'action et sa relation avec la connaissance et les théories émergentes.

Partant de la vision de l'enseignement depuis une conception de la connaissance liée à l'action, montrant le référent que connaissent les professeurs et la manière dont ils arrivent à le connaitre, on avance quelques catégories de base qui doivent être considérées dans le processus d’analyse, telles que les pensées durant l’enseignement interactif, les croyances et théories du professeur. 


\section{Mots-clé}

Connaissance professionnelle, pensées du professeur, complexité, epistémologie, action

\section{Introducción}

"La mayor aportación del conocimiento del siglo XX ha sido el conocimiento de los límites del conocimiento. La mayor certidumbre que nos ha dado es la de la imposibilidad de eliminar ciertas incertidumbres, no sólo en la acción sino también en el conocimiento". Morin, 1999, p. 71.

Este artículo muestra un camino de reflexión que se inicia desde la propuesta investigativa dentro de la línea del pensamiento del profesor, la cual parte de la concepción de un sujeto epistémico que, desde el cultivo sociocultural, ve el "orden" como un aspecto del proceso de conocimiento y no como su único principio; en este sentido, conocer es co-nacimiento, y dentro de este la complejidad es una manera de construir de otra forma.

En este contexto, las acciones humanas son conductas acompañadas de sentido -entendido este como conciencia construida en una serie de procesos de interacción- $y$, por ende, la educación y la función de los profesores se orientarán a la promoción de la existencia de un determinado tipo de sujeto. Lo anterior significa que en dicho contexto se deben establecer mediaciones en los órdenes político, social y cultural que permitan la producción de un tipo de sujeto capaz de provocar la ruptura de esa visión de principios universales, eternos, inmutables, como principios de la 'razón'.

Abordamos la visión de la enseñanza desde una concepción de conocimiento ligado a la acción, que supera la posición de actividad técnica, y mostrado por algunas de las investigaciones que tocan, desde el campo de la formación del profesor, lo referente a qué conocen los educadores, cómo llegan a conocerlo y cómo podrían mejorar ese conocimiento.

Emprender un análisis desde estas categorías es una manifestación del reto que para la educación significa vivenciar, realizar la complementariedad entre los diversos saberes, discursos y, sobre todo, permitir la existencia de distintas visiones de mundo de quienes conforman un pueblo y se identifican como una nación (Huergo, 2003, p. 5): "En algún o algunos aspectos, el sujeto se siente como perteneciendo a una identidad colectiva, que 
lo interpela [...] pertenencia a un nosotros y distinción de otros [...] atributos comunes [...] narrativa histórica común [...] proyecto común”.

El conocimiento del profesor, desde este prisma, obedece por tanto a "un conocimiento de tipo práctico mucho más amplio, que involucra principios construidos y/o interiorizados por el profesor, durante su historia personal y profesional" (Perafán, 2004,p. 24). Esta nueva mirada a la comprensión del profesor responde a los desarrollos en múltiples planos de las ciencias naturales y sociales, en las que las prácticas, las conductas, las conversaciones, los contextos -es decir, la interacción de los individuos y los ambientes- posibilitan recrear comprensivamente los significados y sentidos que el mundo tiene para los actores.

De allí, se parte de la reflexión sobre la línea de investigación del pensamiento del profesor, la visión de reflexión en la acción, el análisis sobre el conocimiento y la epistemología del profesor o dispositivo o procedimiento.

\section{Reflexión en la acción}

En los últimos veinte años se han hecho toda una serie de planteamientos problemáticos acerca de cómo las personas y las organizaciones aprenden, lo que dio lugar a una sucesión de contribuciones sobre cómo los profesionales y las organizaciones producen conocimientos.

Uno de los autores representativos sobre la problemática con el referente de la diversidad de profesiones es Donald Schön. Este pensador explica cómo los profesionales piensan mientras trabajan, ha buscado explicitar los procesos de pensamiento que se realizan mientras trabajamos y que son parte inseparable de las acciones, y ha desarrollado algunos conceptos valiosos para entender el uso del conocimiento tácito de tipo cognoscitivo.

Según Schön, el pensamiento en la acción puede manifestarse explícitamente en palabras, como secuencias de pensamientos lógicos o analógicos; pero también puede hacerlo bajo la forma de sentimientos, impresiones y formación de imágenes y de escenarios subsiguientes; en general, lo que ocurre es una combinación de todos ellos. Mientras actuamos, realizamos una verificación continua entre los resultados deseados y los obtenidos, y hacemos correcciones en el curso mismo de las acciones; esas correcciones pueden operar sobre nuestras acciones sin afectar los supuestos teóricos, o pueden involucrarlos explícitamente: 
Utilizaré el término conocimiento en la acción para referirme a los tipos de conocimientos que revelamos en nuestras acciones inteligentes, ya sean observables al exterior -ejecuciones físicas, como el acto de montar en bicicleta- o se trate de operaciones privadas, como es el caso de un análisis instantáneo de un balance general de una empresa. En ambos casos, el conocimiento está en la acción. Lo revelamos a través de nuestra ejecución espontánea y hábil; y paradójicamente, somos incapaces de hacerlo explícito verbalmente (Schön, 1987, p. 35).

Por lo tanto, el profesional reflexivo puede revisar y criticar no sólo sus acciones, sino también algunos elementos de su teoría en uso y elaborar nuevos elementos, con el fin de hacerla más acorde con el fenómeno que está siendo intervenido. Para lograrlo, es indispensable que la persona tenga en mente la teoría que utiliza y que sepa cómo proyectar los hallazgos en los elementos clave de su teoría en uso; cabe señalar entonces que la calidad de las acciones del profesional dependerá en forma crítica de la calidad de ese pensamiento en la acción.

Podemos afirmar que es así como los seres humanos, en sus relaciones personales, diseñan su comportamiento y sostienen teorías para justificarlo; estas teorías de la acción -en palabras de Schön- contienen valores, estrategias y supuestos que dan cuenta de patrones individuales de comportamiento que funcionan en dos niveles: teorías explícitas (justifican o explican nuestro comportamiento) y teorías implícitas en los patrones de comportamiento espontáneo que mantenemos con los demás.

Este marco presentado permite pensar en un profesional específico: el profesor. La docencia no escapa a los principios de la reflexión en la acción ni a la formación profesional permanente, y esto nos exige preguntar: ¿afectan estos cambios a los profesores? ¿Debemos repensar el trabajo del profesor en estas nuevas circunstancias? ¿Cómo adecuar los conocimientos y las actitudes del profesor para dar respuesta y aprovechar las nuevas oportunidades que la sociedad nos ofrece?

En primer lugar, los cambios solicitados a los profesores no pueden hacerse al margen de cómo se comprende el proceso de aprendizaje de los mismos, de cómo se genera, transforma y transmite el conocimiento en la profesión docente:

Es más, cuando en el mundo de las empresas se está hablando de la necesidad de gestionar el conocimiento como medio para rentabilizar ese saber hacer que los 
empleados han ido acumulando a lo largo del tiempo, en la enseñanza, en palabras de D. Hargreaves, los profesores "ignoran el conocimiento que existe entre ellos; por tanto, no pueden compartir y construir sobre este conocimiento. Al mismo tiempo, tampoco conocen el conocimiento que no poseen y por tanto no pueden generar nuevo conocimiento. Hay una compleja distribución social del conocimiento en la escuela: ningún profesor en particular conoce o puede conocer la totalidad del conocimiento profesional que los profesores poseen" (1999, p. 124). Esto se debe a que gran parte del conocimiento de los profesores es tácito, difícil de articular, y el objetivo de la gestión del conocimiento consiste en ayudar a la organización a utilizar su propio capital intelectual (Marcelo, 2002).

Hoy se demanda un profesor entendido como "un trabajador del conocimiento", y por tanto una profesión docente caracterizada por lo que Shulman (1998, p. 521) ha denominado una comunidad de práctica a través de la cual "la experiencia individual pueda convertirse en colectiva”, una profesión que necesita cambiar su cultura profesional, marcada por el aislamiento y las dificultades para aprender de otros y con otros; esta visión implica acentuar la idea de que la responsabilidad de la formación recae cada vez más en los propios profesionales y en la consolidación de un tejido profesional a través del uso de las redes de profesores.

En este sentido, no se puede seguir esperando que la formación profesional inicial dote al profesor de un bagaje de conocimientos del que pueda disponer a lo largo de toda la vida profesional, sino por el contrario: tanto por la aparición constante de nuevas ocupaciones y profesiones como por el imparable avance de los conocimientos, se requiere una actitud de permanente aprendizaje.

De la misma forma, esa visión de una profesión docente con déficit de consideración social (no se reconoce la labor docente como similar a las profesiones liberales), basada según algunos en las características específicas de las condiciones de trabajo que la asemejan más a ocupaciones que a 'verdaderas' profesiones como la medicina o el derecho, no puede continuar vigente:

Se ha querido comparar sistemáticamente a la docencia con estas otras profesiones, para ver si cumple las condiciones de "un conjunto de individuos que aplican un conocimiento científico avanzado para proporcionar un servicio a los clientes y se agrupan juntos mediante la pertenencia a un cuerpo profesional que asume la res- 
ponsabilidad de controlar los promedios profesionales, y que les confiere beneficios y puede imponer sanciones a los miembros" (Tomlinson, 1996). Y evidentemente, como Hoyle y John (1995) mostraban, la profesión docente, por sus especiales características, no cumple con estos estrictos y clasistas criterios (Marcelo, 2002).

Son precisamente las consideraciones anteriores las que hacen vigente la advertencia de Schön (1987, pp. 268-270) en referencia a las instituciones formadoras de profesionales y a las dependencias de las mismas:

En relación con la universidad, un centro de preparación de profesionales debe enfrentar con su herencia vebleniana ${ }^{1}$ : la actitud de los profesores pertenecientes a los grandes departamentos, igual que la de aquellos pertenecientes a estos centros, para considerarlos como "centros de inferior categoría" completamente dedicados a aplicar la investigación fundamental que se deriva de los "centros superiores", predominantemente dedicados al desarrollo de las disciplinas [...] mi diseño para un centro de preparación de profesionales coherente sitúa un practicum reflexivo en su mismo centro, como un puente entre los mundos de la universidad y de la práctica.

La investigación sobre el pensamiento del profesor parte de un conjunto de supuestos básicos desde los cuales pretende abordar la problemática; entre estos cabe mencionar: la visión del profesor como sujeto reflexivo y racional, capaz de tomar decisiones, emitir juicios, poseer creencias y generar rutinas; considerar que sus pensamientos inciden e incluso determinan su conducta en el aula, y convenir que la reflexión y el pensamiento del docente se entienden desde las dimensiones explícita e implícita.

Es necesario precisar que la línea de investigación sobre el pensamiento del profesor ha sufrido transformaciones como producto de diferentes visiones, que, como en el caso de la psicología, han variado (crítica al conductismo y propuesta cognitiva), y dentro de esta, la comprensión de conducta, de enseñanza, de acción, y muy particularmente el concepto de procesos mentales superiores; la epistemología, con su visión universalista y de unidad, que excluía la subjetividad. se fundamenta en la autoridad de las ideas expresadas por personas con amplia reputación y que con el paso del tiempo no se cuestionan. Es cuando las ideas se institucionalizan al exponerse con gran relieve, sin consultar otras posibilidades. 
Desde este referente, algunos investigadores sistematizan la evolución de la línea como transición entre dos paradigmas, trazado que se presenta de manera precisa por el profesor Perafán (2004, p. 47):

En efecto, ellos plantean que hay dos enfoques que deben tenerse en cuenta al momento de comprender el desarrollo de la investigación sobre el pensamiento del profesor. En primer lugar, el cognitivo, que agrupa los trabajos sobre las operaciones mentales de los profesores, en los distintos momentos de su acción pedagógica, realizados a partir de una marcada influencia de las psicologías cognitivas. En segundo lugar, el alternativo, que se estructura desde la relación analítica y crítica con principios de la teoría social (sociología del conocimiento) y específicamente de la pedagogía (teoría crítica de la enseñanza). En esta última orientación, los estudios más recientes en Sevilla han tomado como punto de referencia la epistemología de la complejidad desarrollada por Morin y, a mi juicio, planteada en gran parte por Gastón Bachelard; además, el grupo de Sevilla ha incorporado a sus estudios los desarrollos de la teoría crítica y el constructivismo. Estas últimas posturas epistemológicas buscan desarrollar el compromiso y la participación de los profesores en la ampliación de los sentidos sociales e históricos de sus prácticas.

\section{¿Qué piensa el profesor? ¿Por qué piensa el profesor?}

Afrontar el problema del conocimiento del profesor implica comprender que la autoobservación es inseparable de lo observado; las autocríticas, inseparables de las críticas; los procesos reflexivos, inseparables de los procesos de objetivación; asimismo, partir del hecho de que hay necesidad de plantearse interrogantes fundamentales sobre el mundo, el hombre y el conocimiento mismo, desde el referente de las condiciones bioantropológicas, socioculturales y de las teorías abiertas. Esto implica que el conocimiento del conocimiento conlleva la integración del sujeto en su conocimiento, debe aparecer ante la educación como un principio y una necesidad permanente que exige una comprensión del sentido del mismo:

Hay dos comprensiones: la comprensión intelectual u objetiva y la comprensión humana intersubjetiva. Comprender significa intelectualmente aprehender en conjunto, com-prehendere, asir en conjunto (el texto y su contexto, las partes y el todo, 
lo múltiple y lo individual). La comprensión intelectual pasa por la inteligibilidad. Explicar es considerar lo que hay que conocer como un objeto y aplicarle todos los medios objetivos de conocimiento. La explicación es obviamente necesaria para la comprensión intelectual u objetiva. La comprensión humana sobrepasa la explicación. La explicación es suficiente para la comprensión intelectual u objetiva de las cosas anónimas o materiales. Es insuficiente para la comprensión humana. Esta comporta un conocimiento de sujeto a sujeto (Morin, 1999, pp. 1-2).

Son estos lineamientos los que orientan contemporáneamente la discusión sobre la visión compleja del conocimiento en general y el del profesor en particular. Autores como Connelly y Clandinin (1984), Porlán y Rivero (1988), Marcelo (1991), Badillo y Azcárate (2002), Moreno (2002), entre otros, han presentado diferentes posturas acerca del estudio sobre el conocimiento del profesor, donde muestran de diversas formas la vigencia de la discusión y la necesidad de seguir desentrañando su red problemática desde una dimensión en la que se considere como contenido en relación con el pensamiento, para que implique descubrir esa dimensión compleja de estructuras teórico-prácticas, integradas, yuxtapuestas..., siendo precisamente la perspectiva contemporánea con elementos de Porlán y Rivero, Assoun y la perspectiva del pensamiento complejo quienes facilitan mediaciones de comprensión de los alcances en la investigación sobre el pensamiento y el conocimiento del profesor.

Por lo tanto, la visión de concebir el conocimiento del profesor como construido en interacción con las experiencias anteriores y actuales, práctico, contextualizado y social, permite abrir la dimensión epistemológica desde una postura de la actividad de enseñanza como un ejercicio de un tipo especial de conocimientos, con el cual se pretende, a su vez, promover otros determinados conocimientos, desde diversas concepciones sobre el mismo y visualizando cómo aprenden los sujetos. Es decir, la consecuencia de pensar en las características generales del conocimiento de los profesores implica incluir su vinculación a contextos, experiencias, tronco social e histórico, la toma de decisiones responsables y las interacciones que modifican una práctica, por lo cual se hace necesaria una sistematización:

[...] convenimos con Marcelo (1993), a quien seguimos en la exposición de sus planteamientos, que las investigaciones desarrolladas por sus colaboradores reducen a cuatro las componentes de este conocimiento en las que se organizan los saberes del profesor: conocimiento de la materia, que incluye tanto su contenido como el de su estructura sintáctica y semántica; el conocimiento pedagógico general, compuesto por los conocimientos sobre los alumnos y el aprendizaje, la gestión de 
clase y el currículum y la enseñanza; conocimiento de contexto, que hace referencia al dónde (la comunidad, el barrio, la escuela) se enseña y a quién (el alumno); el conocimiento didáctico del contenido, que comprende las concepciones del profesor sobre para qué enseña una materia, el conocimiento de las dificultades de su comprensión por los alumnos, el conocimiento del currículum sobre tal disciplina, así como de las estrategias de enseñanza de la misma. Es este último conocimiento el que se ha identificado con las didácticas específicas y, por tanto, el que más se ha trabajado desde estas para valorar la contribución de las mismas al desarrollo del conocimiento profesional; no obstante, según este autor, este conocimiento se construye a partir del que el profesor posee sobre el contenido, así como del conocimiento pedagógico general y el conocimiento de los alumnos. Es precisamente el modelo de razonamiento y acción pedagógica que plantea en relación con la transformación del contenido en materia enseñable una de sus principales aportaciones, ya que distingue en él cinco fases que median desde la planificación hasta la enseñanza misma de un contenido: conocimiento comprensivo de la materia en concreto que se planifica; transformación del contenido que se va a desarrollar, fase de especial importancia que podríamos identificar con la llamada "transposición didáctica"; la enseñanza propiamente dicha, que incluye conocimiento referido a la gestión de clase, presentación de la información, interacción con los alumnos, etc.; la evaluación de la comprensión de los estudiantes y de la propia actuación del profesor; la reflexión, en la que el profesor revisa, reconstruye y analiza críticamente su propia actuación en clase (Estepa, 2000, p. 3).

En este sentido, una propuesta de integración de los distintos componentes del conocimiento del profesor, delineado por el proceso investigativo, permite, por una parte, determinar cuatro características comunes: el conocimiento del profesor se construye, su construcción se da en interacción social y tiene un carácter contextualizado y distribuido; y por otra -de acuerdo con los avances de Porlán y Rivero (1998)-, buscar la integración de cuatro componentes (Perafán, 2004,p. 62): los saberes académicos; los saberes basados en la experiencia; rutinas y guiones, y las teorías implícitas.

Para el efecto, es necesario considerar unos filtros o lentes desde los cuales los profesores toman las decisiones profesionales, y que muestran el hecho de no estar integrados, a partir de unos principios fundantes presentes simultáneamente en el sujeto: los mundos cotidianos de la vida académica, de la vida profesional y de la vida ordinaria. Solamente si se logra el surgimiento de este conocimiento y la apropiación por parte del sujeto profesor, estaremos en camino a una dimensión compleja del mismo. 


\section{Conocimiento - epistemologías emergentes}

El curso actual de nuestra existencia se caracteriza, entre otros aspectos, por dos fenómenos fundamentales: el primero, la crisis de las respuestas totalizadoras, la crisis de las certezas; y el segundo, la rápida obsolescencia de los paradigmas interpretativos de nuestras realidades.

De allí se deriva una consecuencia: la crisis de la disciplinariedad, que por ende implica la apuesta por lo pluridimensional; es decir, el punto de partida de una interpretación acerca de los saberes que poseen los distintos campos frente a un mismo campo o problema.

La reflexión nos lleva a plantear que en las últimas décadas la epistemología no sólo ha ampliado sus perspectivas, sino que se ha transformado radicalmente y ha pasado de la unidad a la dispersión; en otras palabras, la epistemología no es sustentada por ningún consenso, sino que más bien consiste en una especie de red heterogénea de temas, problemas, enfoques, métodos y autores que conviven sin que exista hegemonía por parte de alguno de ellos.

A partir de 1990 y durante la primera década del siglo XXI, ha tenido lugar un apreciable cambio de énfasis en buena parte del pensamiento epistemológico-social contemporáneo; ha ido conformándose una significativa tendencia hacia la que se denomina como una epistemología 'de la complejidad'.

En el desarrollo epistemológico, la denominada epistemología del positivismo, centrada en la relación sujeto-objeto, reduce el primero a un sujeto lógico-metodológico, es decir, de operaciones lógicas y/o metodológicas universales, objetivado (es decir, incapaz de 'contaminar' de subjetividad al objeto indagado), lo que convierte esa relación en una relación entre dos 'objetividades', donde se representan -cuando no incluso se reflejan- las realidades indagadas 'en toda su plena objetividad'.

De acuerdo con estos conceptos, el ser humano se ha preguntado por el qué sabemos y el cómo lo sabemos; siendo el problema epistemológico -es decir, cómo adquirimos el conocimiento de la realidad, si es 'seguro y verdadero' y por tanto, desde un punto de vista muy general, se afirma que es útil- relevante si resiste al mundo de la experiencia y nos capacita para hacer predicciones, o que ciertos fenómenos ocurran o no. Sin embargo, las visiones contemporáneas se han distanciado radicalmente de las tradiciones epistemológicas que se definieron en torno a lo observado y trabajan sus ideas dando forma a las nuevas epistemologías del observador. 
El proceso se encuentra con los planteamientos de Morin (1999: UNESCO, cap. V: p. 5) y la teoría de la complejidad; el autor nos plantea que el conocimiento pertinente debe enfrentar la complejidad, puesto que es

en las incertidumbres doctrinales, dogmáticas e intolerantes donde se encuentran las peores ilusiones; en cambio, la conciencia del carácter incierto del acto cognitivo constituye la oportunidad para llegar a un conocimiento pertinente, el cual necesita exámenes, verificaciones y convergencia de indicios; así, en los crucigramas, se llega a la precisión por cada palabra adecuada según su definición y su congruencia con las otras palabras que abarcan letras comunes; la concordancia general que se establece entre todas las palabras constituye una verificación de conjunto que confirma la legitimidad de las diferentes palabras inscritas. Pero la vida, a diferencia de los crucigramas, comprende casos sin definición, casos con falsas definiciones y, especialmente, la ausencia de un marco general cerrado; es sólo allí donde se puede aislar un marco y se pueden manejar elementos clasificables, como en el cuadro de Mendeleïev, que se pueden lograr certezas. Una vez más, repitámoslo: el conocimiento es navegar en un océano de incertidumbres a través de archipiélagos de certezas.

Esta propuesta nos lleva a un cambio de mirada, a una nueva forma de pensar: pensar sistemáticamente, en donde la posición del observador -su ángulo de mira- modifica lo observado y determina la naturaleza de la observación. Necesitamos un sujeto que sea a la vez unidad / multiplicidad / totalidad / diversidad / organización, porque la simplificación aísla en relación no sólo con su entorno, sino con otros sistemas, con el tiempo, con el observador / conceptuador, y además deifica; es decir, oculta la relatividad de las nociones de sistema, subsistema, suprasistema... En últimas, disuelve la organización y el sistema.

La contraposición es lo pluridimensional, como primera aproximación. Las regiones constituyen la recontextualización de las disciplinas en grandes unidades, que operan tanto en el campo intelectual de las disciplinas como en el de las prácticas. De acuerdo con Bernstein, lo que se presencia es un movimiento hacia la regionalización del conocimiento. Hasta cierto punto, se podría decir que en las regiones hay una base interdisciplinaria, que hay un discurso interdisciplinario y que el tipo de interdisciplinariedad es constitutivo (expresa la articulación, la relación o el diálogo de las disciplinas).

Hoy -en términos de Morin- el mundo se concibe desde el ámbito de los sistemas complejos, se torna inestable, fluctúa entre el orden y el caos -caos significaba desorden y 
confusión; actualmente significa clases de orden complejas, ultrasensibles y sutiles-, y demanda de las ciencias de la sociedad, preocupación por el contexto cultural y social en que viven; nos pide conocer la identidad humana, nos propone una antropología compleja que reconozca las cualidades de racionalidad e irracionalidad del ser humano y su vinculación entre individuo-especie-sociedad-cultura; optar por una mirada transdisciplinar, de este modo integradora, que permita así penetrar su complejidad:

Hay una relación de tríada individuo-sociedad-especie. Los individuos son el producto del proceso reproductor de la especie humana, pero este mismo proceso debe ser producido por dos individuos. Las interacciones entre individuos producen la sociedad y esta, que certifica el surgimiento de la cultura, tiene efecto retroactivo sobre los individuos por la misma cultura. No se puede absolutizar al individuo y hacer de él el fin supremo de este bucle; tampoco se lo puede aislar de la sociedad o de la especie. A nivel antropológico, la sociedad vive para el individuo, el cual vive para la sociedad; la sociedad y el individuo viven para la especie, la cual vive para el individuo y la sociedad. Cada uno de estos términos es a la vez medio y fin: son la cultura y la sociedad las que permiten la realización de los individuos y son las interacciones entre los individuos las que permiten la perpetuidad de la cultura y la autoorganización de la sociedad. Sin embargo, podemos considerar que la plenitud y la libre expresión de los individuos-sujetos constituyen nuestro propósito ético y político sin dejar de pensar también que ellos constituyen la finalidad misma de la tríada individuo-sociedad-especie. La complejidad humana no se comprendería separada de estos elementos que la constituyen: todo desarrollo verdaderamente humano significa desarrollo conjunto de las autonomías individuales, de las participaciones comunitarias y del sentido de pertenencia con la especie humana (Morin, 1999).

En consecuencia, diferentes investigadores en distintas disciplinas y regiones comienzan a abordar de otra manera fenómenos que hasta entonces eran inexplicables para el ser humano, por no hallarles ninguna pista de orden, sentido, coherencia. El supuesto fundamental de la teoría del caos es que la unidad individual no importa; lo que importa son las simetrías recursivas entre los diferentes niveles del sistema. El caos se constituye como ciencia al hallar entonces ciertos reguladores de largo plazo, cierta simetría de conjuntos, atractores y nodos, los cuales configuran leyes que de allí en adelante permiten trabajar con el azar, con lo inestable, lo disipativo y lo incierto. 


\section{Conclusiones}

Estas reflexiones nos llevan a la necesidad de reconocer la participación activa del sujeto en la construcción del conocimiento, asumiendo que los actos de explicar (análisis cuantitativo) o comprender (interpretación cualitativa) no relegan al sujeto a una posición pasiva de representación del mundo, sino que lo incluyen como co-constructor de la 'realidad.'De la misma forma, los dos enfoques son la expresión de las tradiciones explicativa -establece relaciones de causalidad desde una metodología cuantitativa- y comprensiva -establece los significados y sentidos que las personas atribuyen a los fenómenos de su discurrir diario, desde una metodología cualitativa-; disputa excluyente que en procura de ser superada posibilita la propuesta de un modelo que responda al principio dialógico del pensamiento complejo, que asuma la concurrencia y la complementariedad de los opuestos, y no su mutua exclusión.

Es en este sentido como de una u otra forma nos encontramos con el abordaje del problema del conocimiento, cuestionamiento que nos remite a comprender la existencia de una pluralidad de epistemologías con sus correspondientes opciones metodológicas, las cuales nos plantean la urgencia de concebir las que tienen por centro lo observado y las que se centran en el observador que observa observadores, observación activa sobre prácticas, conductas, conversaciones, narrativas, contextos (Pérez, 2004, p. 1):

Los contundentes cuestionamientos de los fundamentos tradicionales de la validación del conocimiento científico que inició Popper en la primera mitad del siglo $\mathrm{XX}$, así como los postulados de Einstein, Heisemberg y Planck, quienes reclamaron la relatividad y la incertidumbre del mundo de la microfísica, además de las propuestas de Von Foerster sobre la cibernética de segundo orden, de Bateson sobre la unidualidad del cuerpo y de la mente, de Heidegger acerca del carácter preformativo del lenguaje, de Maturana y Varela sobre la biología del conocimiento, hasta llegar a las propuestas múltiples del pensamiento complejo de Morin y Capra, entre otros, son visiones que han ampliado el panorama de las maneras de hacer ciencia.

Lo anterior nos permite evidenciar la importancia de la participación activa del sujeto en relación con la construcción del conocimiento, en tanto que como artífice del mismo se convierte en un co-constructor de la realidad. Entre otros, estos presupuestos son, indispensablemente, los que debemos entrar a considerar en los procesos, orígenes y desarrollo de la investigación acerca de los procesos de pensamiento del profesor: 
Históricamente, el profesor ha pasado de ser comprendido en términos de sus conductas docentes eficientes, a serlo como un sujeto que piensa y que con sus pensamientos media sus acciones. El profesor de ciencias, como intelectual o como investigador, lo es en cuanto puede dar cuenta de los efectos históricos de su posicionamiento en la cultura (Perafán, 2004, p. 40).

Por lo tanto, investigar sobre el pensamiento del profesor se convierte en un reto que parte de esa visión de ser humano que nos aparece en su complejidad:

Es un ser a la vez totalmente biológico y totalmente cultural. El cerebro por medio del cual pensamos, la boca por medio de la cual hablamos, la mano por medio de la cual escribimos, son órganos totalmente biológicos al mismo tiempo que totalmente culturales [...] En lo sucesivo, el concepto de hombre tiene una doble entrada: una entrada biofísica y una entrada psico-sociocultural, y las dos entradas se remiten mutuamente (Morin, Conferencia: Sujeto Humano y Conocimiento).

También urge realizar la ruptura con la visión que concibe al profesor como un técnico aplicador del currículo y cuyas funciones son dominar y reproducir un amplio repertorio de destrezas consideradas eficaces, con el fin de posibilitar la dimensión sobre los pensamientos y las acciones del profesor bajo la premisa de unos supuestos novedosos: somos sujetos reflexivos, racionales, y por tanto tomamos decisiones, planteamos juicios, tenemos creencias y rutinas propias a nuestra formación profesional; por ello, nuestros pensamientos influyen en el comportamiento, en el acontecer del aula. En consecuencia, el pensar y conocer del maestro tiene dos tópicos: uno de fácil acceso y otro implícito o tácito, que exigen el uso de instrumentos diferentes para cada caso.

Por tanto, solamente si nos comprendemos como seres socioculturales, la dimensión de la interacción, y por ende la interrelación, adquieren una real trascendencia: somos seres mediatizados culturalmente, y la cultura debe visualizarse como texto complejo (existencia extrasubjetiva e intrasubjetiva), objeto de intervención para la educación, expresado en una de sus conferencias por el profesor Rafael Ávila:

La reforma del pensamiento necesaria es aquella que generará un pensamiento del contexto y de lo complejo. El pensamiento del contexto: se trata de buscar siempre la relación de inseparabilidad y de ‘interretroacción’ entre todo fenómeno 
y su contexto, y de todo contexto con el contexto planetario. El pensamiento de lo complejo: es necesario un pensamiento que capte las relaciones, las interacciones y las implicaciones mutuas, los fenómenos multidimensionales, las realidades que son a la vez solidarias y conflictivas, que respeta lo diverso, toda vez que reconoce la individualidad; un pensamiento organizador que concibe la relación recíproca entre el todo y las partes.

Asimismo, se da apertura al horizonte sobre la educación, ya que posibilita el abordaje de su problemática desde diversas miradas: la educación debe contribuir a la autoformación de la persona, pues esta debe aprender a asumir la condición humana, aprender a vivir y aprender a convertirse en un ciudadano; por lo tanto, los maestros, en palabras de Morin (2001), conforman una comunidad de destino: "La comunidad es de carácter cultural/ histórico. Es cultural por los valores, costumbres, ritos, normas, creencias comunes; es histórica por los avatares y pruebas sufridas en el curso del tiempo".

Esto significa la presencia del principio de intersubjetividad, de la interpretación/evocación, el privilegio de la lógica del descubrimiento y la invitación a trabajar en la organización de la relación con la 'realidad' como proceso de apropiación, y no sólo como correlato que requiere ser sometido a contrastación. Lo investigado se asume ya no como descubrimiento, sino como construcción en el diálogo de la tensión entre lo que el profesor es y lo que hace, y ese mundo que pretende conocer.

Es necesario, por lo tanto, comprender que la visión de mundo de cada profesor y su manera de actuar en él no caen en un reduccionismo, y que, por el contrario, de acuerdo con Perafán (2004, p. 96):

[...] los conocimientos de los sujetos (profesores) se forman de manera significativa por la asimilación de los nuevos contenidos a las ideas más inclusivas y relacionadas, establecidas en la mente, y que en esa asimilación los conceptos establecidos pueden ser modificados; podemos presumir (y con seguridad evidenciar) que los vínculos entre los conceptos de diferentes campos en el espacio de las tendencias propias de cada profesor generan verdaderos sistemas complejos de pensamiento y de conocimiento.

El proceso de estudio del pensamiento del profesor nos permite atisbar la posibilidad de entender la epistemología del profesor como un concepto complejo, que a su vez permite 
reconocer los rasgos característicos del tipo de conocimiento que constituye a cada uno de ellos, desde la asimilación de lo cultural por parte del sujeto. Podemos afirmar que el conocimiento del docente es flexible, conflictivo, incierto, y que permite identificar un perfil epistemológico diverso que involucra el conocimiento disciplinar, el conocimiento enseñado y la imagen que ha construido gracias a la variedad de principios, normas y reglas diversas: "[...] queda clara la necesidad de entender la epistemología de los profesores de acuerdo con cada dominio y campo del conocimiento y, seguramente, según cada uno" (Perafán, 2004, p. 100).

A manera de conclusión, se puede afirmar que en la actualidad es necesario reflexionar sobre la forma en que se enseña, los sujetos que enseñan, a quienes se enseña y, lo que es más importante, cómo se ha llegado a conocer lo que se enseña y su relación con el contexto.

\section{Referencias}

Badillo, R.E. \& Azcárate, C. (2002). Conocimiento profesional del profesor de matemática: integración del conocimiento del contenido matemático y el conocimiento didáctico del contenido. En: Perafán G.A. y Adúriz Bravo, A. (2002). Pensamiento y conocimiento de los profesores. Debate y perspectivas internacionales. Bogotá: UPN-Colciencias.

Connelly, F.M. \& Clandinin, D.J. (1984). Personal Practical Knowledge at Bay Street School: Ritual, Personal Philosophy and Image. En Halkes, R. y Olson, J.K. (eds.). Teacher Thinking. A New Perspective on Persisting problems in Education. Lisse: Sweets and Zeitlinger, pp. 134-148.

Estepa, G.J. (2000). La investigación sobre el conocimiento profesional de los profesores para enseñar Ciencias Sociales. Universidad de Huelva. Ponencia XI Simposio Internacional de Didáctica de las Ciencias Sociales.

Huergo, J. (2003, junio). Lo que articula lo educativo en las prácticas socioculturales. Instituto de Cultura Popular.

Marcelo, C. (1987). El pensamiento del profesor. Barcelona: Ceac.

Marcelo, C. (1991). El estudio de caso. Una estrategia para la formación del profesorado y la investigación didáctica. En Marcelo G.C., et ál. (eds.). El estudio de caso en la formación del profesorado y la investigación didácticas. ISP de la Universidad de Sevilla, pp. 11-73. 
Marcelo, C. (2002, agosto 16). The Education Policy Analysis Archives. Aprender a ensenar para la sociedad del conocimiento. Volumen 10. No. 35. Universidad de Sevilla.

Maturana, H. (1995). La realidad: ¿objetiva o construida? Vol. I y II. Barcelona: Editorial Anthropos, Universidad Iberoamericana e ITESO.

Moreno, M. (2002). El pensamiento del profesor. Evolución y estado actual de las investigaciones. En Perafán G.A. y Adúriz Bravo, A. (2002). Pensamiento y conocimiento de los profesores. Debate y perspectivas internacionales. Bogotá: UPN-Colciencias.

Morin, E. (1999). El método: el conocimiento del conocimiento. Madrid: Ediciones Cátedra S.A.

Morin, E. (1999). Los siete saberes necesarios a la educación del futuro. París, Francia: Organización de las Naciones Unidas para la Educación, la Ciencia y la Cultura.

Morin, E. (2001). Los desafíos. Copias Conferencia ICFES.

Morin, E. (2004). Introducción al pensamiento complejo. Barcelona: Gedisa Editorial.

Perafán E., G. (2004). La epistemología del profesor sobre su propio conocimiento profesional. UPN.

Perafán E., G. \& Arduriz - Bravo, A. (2002). Pensamiento y conocimiento de los profesores. Bogotá: UPN - Colciencias.

Pérez P., T. (2004). La perspectiva constructivista en la investigación social. Fotocopias Seminario, UPN.

Porlán, A.R. \& Rivero, G.A. (1998). El conocimiento de los profesores. Sevilla: Díada.

Shón, D.A. (1987). La formación de profesionales reflexivos. Madrid España: Paidós.

Shulman, L. (1998). Theory, Practice, and the Education of Professional. En The Elementary School Journal, 98, (5), 511-526. 